\title{
Comparison of liaison psychiatry service models for older patients
}

\section{AIMS AND METHOD}

At a London teaching hospital, the existing off-site consultation model psychiatric liaison service for older people was replaced with an on-site liaison model service in December 2000. Several indicators of the functioning of the service were audited using identical methods before and after this change.

\section{RESULTS}

The case-load increased by $50 \%$, but the liaison psychiatrists were more satisfied with the appropriateness of referrals. The case mix did not change. The new service achieved target waiting times more consistently, particularly for urgent referrals. Referring teams were more satisfied with the speed of response, while the new service maintained the salience and clarity of advice.

\section{CLINICAL IMPLICATIONS}

Findings are on the whole favourable, and support the wider introduction of specialist old-age liaison psychiatric services.
Liaison psychiatrists need special skills to assess and deliver quality care to older patients, where comorbidity with multiple physical and cognitive impairments is common. Adjustment reactions to hospitalisation, treatment and loss of independence might be particularly profound (Goldberg, 1989). Special knowledge is required in three main areas: the laws that safeguard the rights of older people who are vulnerable (e.g. power of attorney, court of protection, guardianship); clinical ethics and the complex issues surrounding assessment of capacity in impaired patients (Lederberg, 1997); and the variety of placement options and the network of community supports for effective discharge (Starkman \& Hall, 1979).

Twenty years ago, $30 \%$ of all liaison psychiatry referrals were for people over the age of 65 (Lipowski, 1983); with demographic ageing continuing apace, this proportion will have risen inexorably. However, in the UK, specialist liaison services for older people are the exception. Different models have been described, most of them ad hoc: care provided by the general adult liaison service (Lipowski, 1983); service provision by old-age psychiatry community mental health teams (CMHTs; Scott et al, 1988; De Leo et al, 1989); and collaborative care provided jointly by adult liaison and old-age psychiatry services (Small \& Fawzy, 1988; Kisely \& Axten, 2000). The effectiveness of these different models has not been studied

\section{Method}

\section{Setting and service}

King's College Hospital is a 950-bed London teaching hospital covering a large inner-city catchment area. Last year there were 84500 in-patient admissions, with an average of 37 acute admissions daily. All acute admissions are to the main King's College Hospital site. Patients might be transferred to Dulwich Hospital for rehabilita- tion prior to discharge. The Liaison Psychiatry Service for Older People, covering both sites, was restructured completely in 2000. We moved from an ad hoc off-site service to a fully resourced specialist service, with a dedicated part-time consultant in old-age liaison psychiatry, and a full-time staff grade psychiatrist and a senior house officer trainee based in the two sites. Simultaneously, the old consultation model of service delivery was changed to a true liaison model. Previously, referrals were transmitted by fax to a community psychiatrist based in another hospital, who visited to assess the patient as soon as feasible and entered treatment recommendations in the clinical notes. There was little possibility for direct liaison. In the new services, liaison psychiatrists attend the Health Care of the Elderly physicians' multi-disciplinary management rounds (MDMs), where decisions are made regarding rehabilitation, discharge planning and placement. They are involved in discussions about many of the in-patients, disseminating better mental health awareness and practice throughout the multi-disciplinary team. However, consultations are targeted efficiently at those patients who would most benefit from a psychiatric assessment and intervention. Findings and recommendations are fed back directly at future MDMs. Progress is monitored, and liaison is direct and continuous throughout the admission. Referrals might be initiated by the psychiatrist or by any member of the multi-disciplinary team. The psychiatrist's presence on site also facilitates informal referrals in the intervals between MDMs. With the introduction of the new service, explicit arrangements were made for liaison with local old-age psychiatry CMHTs. Patients known to these services would continue to be managed by them as inpatients, with the support of the liaison team. Patients not known to the CMHT but requiring support on discharge were referred by the liaison team to enable their involvement in discharge planning. For patients without a need for formal CMHT follow-up, information was passed on a 'need to know' basis. 
R

original papers

\section{Audit}

The new service has been audited extensively since its inception, including all patients aged 65 and over seen during a 1-year period between December 2000 and December 2001. The main standards set for the new service were assessment of all urgent referrals within 24 hours and assessment of all medium- and low-urgency referrals within 5 days. Descriptive data were produced using the Statistical Package for the Social Sciences version 11.0 and compared using Chi-squared with data collected in an analogous fashion during a 7-month period in 1998/1999, demonstrating the workings of the old service.

Four forms were completed for each patient:

(1) Referral form - completed by the referring team giving patient details, category of problems and specific questions to be answered;

(2) Initial assessment form - completed by the liaison psychiatrist following the first assessment giving mental state assessment, diagnosis and initial management plan. The psychiatrist also rated their satisfaction with the completeness, appropriateness and clarity of the referral, which was coded on a five-point scale with 1 meaning 'not satisfied at all' and 5 meaning very satisfied

(3) Referrer feedback form - completed by the referring doctor after the initial assessment, who rated their satisfaction in the same way with the speed, appropriateness and clarity of the psychiatrist's response;

(4) Final assessment form - completed by the liaison psychiatrist at the end of their involvement with the patient giving final diagnoses, number of assessments, time spent with patient, outcome and follow-up arrangements.

\section{Results}

\section{Workload}

The new service received 349 requests for assessment during the 1-year period 2000-2001 (excluding the large number of cases only discussed during MDMs, a facility which did not exist under the old service). This constituted 1.4 new referrals per working day, an increase in the rate from 0.95 referrals per working day for the old service (187 referrals in 7 months in 1997/1998). There were no significant differences in patient characteristics between the new and old service (Table 1).

The new service made a total of 674 initial and follow-up assessments, an average of 2.7 patient assessments per working day. Follow-up data were incomplete for the old service, but a greater proportion of patients referred to the new service were seen two or more times (44\%) when compared with the old service (315). The mean patient total contact time was $83 \mathrm{~min}$ (s.d. $62 \mathrm{~min}$ ) for the new, and $73 \mathrm{~min}$ (s.d. $43 \mathrm{~min}$ ) for the old service.
Table 1. Main characteristics of referrals seen by the old and new

liaison service

\begin{tabular}{|c|c|c|}
\hline & $\begin{array}{l}\text { New service } \\
\text { (12 months) }\end{array}$ & $\begin{array}{l}\text { Old service } \\
\text { (7 months) }\end{array}$ \\
\hline Patients seen & 336 & 183 \\
\hline Gender (female) & $224(67 \%)$ & $109(60 \%)$ \\
\hline Age mean years (s.d.) & $81.5(7.4)$ & $81.2(7.1)$ \\
\hline $\begin{array}{l}\text { Admitted from home } \\
\text { Referred by }\end{array}$ & $299(89 \%)$ & $161(88 \%)$ \\
\hline MDM & $148(44 \%)$ & N/A \\
\hline Other & $188(56 \%)$ & \\
\hline \multicolumn{3}{|l|}{ Referral urgency } \\
\hline High & $79(24 \%)$ & $39(22 \%)$ \\
\hline Medium & $227(68 \%)$ & $122(68 \%)$ \\
\hline Low & $29(9 \%)$ & $17(10 \%)$ \\
\hline
\end{tabular}

\section{Case mix}

The case mix of referred patients did not seem to have changed following the restructuring of the service (Table 2). For the old service, this information was available for only $59 \%$ of cases.

\section{The work of the new liaison service}

With the new service an overall $62 \%$ of all referrals were seen within 24 hours. We aimed to see all urgent referrals within 24 hours; we achieved this target for $96 \%$ of urgent referrals, compared with $40 \%$ for the old service $(P<0.001)$. We aimed to see medium-urgency referrals within 5 days. We achieved this target for $98 \%$ of medium-urgency referrals compared with $76 \%$ for the old service $(P<0.001)$. Two-thirds of low-urgency referrals were seen within 5 days, both under the old and new services.

The most frequent specific initial questions for the new service could be classified under mental status assessment ( $50 \%)$, assessment of decision making capacity (20\%) and review of medication (14\%). Liaison psychiatrists were also asked to answer questions about patients' suicidality (6\%) and placement (5\%). Requests for assessment of capacity seem to have grown with the advent of the new service, previously accounting for $10 \%$ of referrals. Initial management also differed between the

Table 2. Primary psychiatric diagnosis

\begin{tabular}{|lcc} 
& $\begin{array}{c}\text { New service } \\
(n=336) \\
\%(n)\end{array}$ & $\begin{array}{c}\text { Old service } \\
(n=108) \\
\%(n)\end{array}$ \\
\hline Dinal primary diagnosis & $33(111)$ & $29.6(32)$ \\
Depression & $33.6(113)$ & $33.3(36)$ \\
Acute confusional state & $7.1(24)$ & $9.3(10)$ \\
Adjustment disorder & $3.3(11)$ & $3.7(4)$ \\
Psychotic or delusional disorder & $6.6(22)$ & $3.7(4)$ \\
Other & $13.9(50)$ & $15.7(17)$ \\
No psychiatric diagnosis & $1.5(5)$ & $4.6(5)$
\end{tabular}


old and the new services. Under the new service, patients were much less likely to be prescribed antidepressants $(12 \%$ v. $28 \%, P<0.001)$. The prescribing rate of antipsychotics was similar ( $8 \%$ v. $7 \%$ ).

\section{Satisfaction}

There were clear increases in levels of satisfaction with the new service by the referring teams for the key indicator of response time, with $96 \%$ reporting net satisfaction (scores of 4 or 5 ) v. $81 \%$ under the old service $(P<0.001)$. The previously high satisfaction ratings for salience of advice from the liaison psychiatrist ( $98 \% \mathrm{v}$. $96 \%$ satisfied) and clarity of advice (96\% v. 94\%) were maintained. Increases were also observed in the satisfaction ratings of the liaison psychiatrist for the appropriateness (86\% reporting net satisfaction v. 74\% under the old service, $P=0.01)$, clarity $(85 \%$ v. $73 \%$, $P=0.01)$ and completeness ( $76 \%$ v. $55 \%, P<0.001)$ of the referrals.

Just over half of the patients seen by the new service returned to their own homes after discharge. A smaller number required residential (15\%) and nursing home $(17 \%)$ placement, and $11 \%$ of patients died. Interestingly, just 3\% required transfer to a psychiatric hospital and only $18 \%$ of patients required $\mathrm{CMHT}$ psychiatric follow-up. Other follow-up arrangements were: general practitioner, $34 \%$; social care, $24 \%$; multiagency, 9\%; and no follow-up, 16\%. Outcome and follow-up data were incomplete for the audit of the old service, therefore direct comparison was not possible.

\section{Discussion}

Three clear messages emerge from this audit. First, the introduction of the new liaison service has reduced waiting times for assessment, particularly for urgent cases with a consequent increase in referrer satisfaction. Second, despite concerns that the accessibility of the new service would lead to it being swamped with referrals (Scott et al, 1988; De Leo et al, 1989), the 50\% increase in rate of referrals has been manageable. The increase in the liaison psychiatrists' satisfaction with the appropriateness of the physicians' referrals suggests improved targeting and efficiency. This finding is consistent with other research, suggesting a decline in the referral rate following the introduction of a liaison model (Swanwick et al, 1994). Third, the high proportion of cases of dementia among the referred patients and the large numbers of requests for assessment of capacity suggest the need for a distinct specialist old-age service. However, the relatively small proprtion of referred patients requiring follow-up by old-age $\mathrm{CMHT}$ services suggests that most problems are specific to the inpatient context, and that this service for older patients is best provided by a dedicated hospital-based liaison team, working closely with CMHT colleagues. Through early recognition and treatment of mental illness among older patients on medical wards, the liaison psychiatry service can help their rehabilitation and improve their prognosis
(Collinson \& Benbow, 1998), thus shortening their stay in hospital (Strain et al, 1991). We have now developed a ward-based protocol to assist the older adult physicians in early identification of capacity issues, which, if unanticipated, might delay discharge significantly.

The interaction between the two services should develop the diagnostic and management skills of each. We are now building on the successful establishment of the new service delivery model, by working with colleagues in Health Care of the Elderly and other disciplines to develop agreed protocols for efficient assessment and management of commonly encountered mental health problems. We will disseminate these protocols through in-service training sessions conducted as part of the existing clinical education programme, to which the Liaison Psychiatry Service for Older People has contributed since its inception. In our opinion, the service offers a unique possibility for training in old-age liaison psychiatry, a sub-discipline which will clearly need to be established in future years, given the rising proportion of general hospital admissions accounted for by older persons.

\section{Declaration of interest}

None.

\section{References}

COLLINSON, Y. \& BENBOW, S. M. (1998)

The role of an old age consultation nurse. International Journal of Geriatric Psychiatry, 13, 159-163.

DELEO, D., BAIOCCHI, A., CIPOLLONE, B., et al (1989) Psychogeriatric consultation within a geriatric hospital: a six year experience. International Journal of Geriatric Psychiatry, 4 135-141.

GOLDBERG, R. L. (1989) Geriatric consultation/liaison psychiatry (Issues in geriatric psychiatry). Advances in Psychosomatic Medicine, 19, 138-150.

KISELY, S. \& AXTEN, C. (2000)

Collaboration between general and old age psychiatrists in the provision of a consultation-liaison service. The Royal Australian and New Zealand College of Psychiatrists 35th Annual Congress. Adelaide, South Australia, 27-30 April 2000.

LEDERBERG, M. S. (1997) Making a situational diagnosis. Psychiatrist at the interface of psychiatry and ethics in the consultation-liaison setting.

Psychosomatics, 38, 327-328.

SCOTT, J., FAIRBAIRN, A. \& WOODHOUSE, K. (1988) Referrals to a psychogeriatric consultation-liaison service. International Journal of Geriatric Psychiatry, 3, 131-135.

SMALL, G.W. \& FAWZY, F. I. (1988) Psychiatric consultation for the medically ill elderly in general hospital: need for a collaborative model of care. Psychosomatics, 29, 94-103.

STARKMAN, M. N. \& HALL, G. G. (1979) Teaching medical gerontology: utilisation of psychiatry consultation program. Journal of Medical Education, 54, 643-648.

STRAIN, J. J., LYONS, J. S., HAMMER, J. S., et al (1991) Cost offset from a psychiatric consultation-liaison intervention with elderly hip fracture patients. American Journal of Psychiatry, 148, 1044-1049.

SWANWICK, G. R. J., LEE, H., CLARE, A. W., et al (1994) Consultation-liaison psychiatry: a comparison of two service models for geriatric patients. International Journal of Geriatric Psychiatry, 9, 495-499.

LIPOWSKI, Z. J. (1983) The need to integrate liaison psychiatry and geropsychiatry. American Journal of Psychiatry, 140, 1003-1005.

*Fedza Mujic Staff Grade in Liaison Psychiatry for Older People, Charlotte Hanlon Specialist Registrar in Psychiatry, Danny Sullivan Specialist Registrar in Psychiatry, GinaWaters Consultant Psychiatrist, Martin Prince Consultant Psychiatrist and Professor of Psychiatric Epidemiology, Department of Psychological Medicine (King's College Hospital), Maudsley Hospital, Denmark Hill, London SE5 8AZ original papers 